\title{
ANÁLISE TEÓRICO-EXPERIMENTAL DA DISPERSÃo DE POLUENTES LÍQUIDOS EM SOLOS
}

\section{THEORETICAL-EXPERIMENTAL ANALYSIS OF LIQUID POLLUTANT DISPERSION IN SOILS}

\author{
JOVANI LUIZ FAVERO \\ Engenharia de Bioprocessos e Biotecnologia (com ênfase ambiental) Universidade Estadual do Rio Grande do Sul, \\ Núcleo de Caxias do Sul
}

ANDRÉ RodRigues MUNIZ

Universidade Estadual do Rio Grande do Sul, Núcleo de Caxias do Sul

Ricardo PaUpitz Barbosa dos Santos

Departamento de Agronomia. Universidade Estadual de Maringá/UEM

Recebido: 27/10/06 Aceito: 23/10/07

\begin{abstract}
RESUMO
Acidentes envolvendo o contato de substâncias químicas tóxicas com o meio ambiente são muito freqüentes. Exemplos são derramamentos acidentais de combustíveis e solventes industriais. Seria interessante predizer a distribuição da substância no solo em função do tempo de contato. Isso possibilitaria prever a extensão do impacto ambiental causado por algum acidente e auxiliar na aplicação de técnicas de remediação. Sendo assim, desenvolveu-se um código computacional para simular a dispersão de poluentes líquidos em solos, a partir de um modelo matemático baseado nos princípios de conservação de massa e transporte de poluentes em meios porosos. Resultados obtidos em simulaçôes foram comparados com experimentos realizados em pequena escala, apresentando uma boa concordância.
\end{abstract}

PALAVRAS-CHAVE: Transporte de poluentes em solos; contaminação de solos; simulação numérica; engenharia ambiental.

\begin{abstract}
Accidents which result in the contact of hazardous chemical substances with the environment are frequent. Typical examples are solvents accidental spills and inadequate waste disposal. Therefore, it would be interesting to predict the distribution of the substance throughout the soil as a function of contact time, in order to foresee the environmental impact and help in the application of remediation techniques. It has been developed a computational code to simulate the dispersion of pollutants in soils, by solving a mathematical model based on the mass conservation and on the transport rate of pollutants in porous media. Numerical results were compared with experimental data, showing a good agreement.
\end{abstract}

KEYWORDS: Pollutant transport in porous media, soil contamination, numeric simulation, environmental engineering.

\section{INTRODUÇÃO}

Nos últimos 200 anos verificou-se o rápido crescimento da população por todo o planeta, resultando na necessidade de quantidades cada vez maiores de combustíveis, desenvolvimento das indústrias químicas e farmacêuticas, de fertilizantes e pesticidas para sustentar e melhorar a "qualidade de vida". Com o crescimento industrial houve também um aumento significativo dos desastres ecológicos (Mesquita, 2004).

Freqüentemente é noticia na TV algum acidente envolvendo derramamento de substâncias químicas, geralmente tóxicas aos seres vivos.
Exemplos disso são: combustíveis, solventes industriais, matéria-prima para a indústria química e outros produtos químicos orgânicos e/ou inorgânicos. Até que esta substância seja removida da superfície ou evapore, a mesma passa muito tempo em contato com a superfície do solo, provocando a sua contaminação (Rajasekaran et al, 2005). Estes derramamentos ocorrem em muitas situações, tais como: vazamentos em tubulações de transporte de fluidos, acidentes envolvendo veículos de transporte de substâncias químicas, perfurações em tanques de armazenamento de combustíveis, resíduos depositados sobre o terreno natural a mercê de chuvas, disposição indevida de resíduos em aterros onde não há impermeabilização adequada do solo, entre outras situações (Edwards, 2002).

A poluição se tornou inaceitável para a sociedade, aumentando a preocupação com os seus efeitos sobre o meio ambiente. Preocupações com a qualidade do ar e das águas são antigas, mas as preocupações com solos contaminados tornaram-se evidentes somente no final da década de 70 (Mesquita, 2004). Os danos provocados pelos poluentes no solo podem ser desastrosos, podendo afetar populaçóes de organismos, comunidades ou mesmo causar graves danos em todo o ecossistema local. 
Infelizmente é impossível reverter todos os danos causados ao ambiente utilizando técnicas de remediação de solos. As estratégias modernas de gerenciamento têm dado ênfase à minimização de resíduos, reciclagem e remediação em preferência à disposição dos resíduos no meio ambiente (Edwards, 2002).

Dependendo da extensão do derramamento de algum poluente e das propriedades físicas do solo e do próprio poluente deve-se considerar também o problema da contaminação das águas subterrâneas. As duas maiores fontes abastecedoras de água para indústrias e cidades são as chamadas águas de superfície (rios, lagos, etc.) e aqüíferos subterrâneos. Este último, principalmente, tem sido contaminado freqüentemente devido a acidentes envolvendo petróleo e seus derivados. O relatório de áreas contaminadas no estado de São Paulo, publicado pela CETESB em maio de 2002, nos dá um retrato da situação do subsolo de uma das regiōes mais industrializadas do país. Dos 255 casos registrados, $60 \%$ estão relacionados a contaminação por combustíveis (gasolina e diesel), $15 \%$ a solventes clorados, $5 \%$ a solventes orgânicos não clorados e $3 \%$ a hidrocarbonetos poliaromáticos. Os outros casos envolvem pesticidas e metais pesados (Mesquita, 2004).

Isto constitui um grave problema, pois as águas subterrâneas são responsáveis por $60 \%$ do consumo de água potável no Brasil, além de tratar-se de um recurso complementar mais barato na área servida pela rede pública de abastecimento, dispensando obras caras de captação, adução e tratamento (Rebouças, 1994). A persistência de xenobióticos orgânicos no ambiente é um problema de importância pública, científica e legislativa, devido ao seu potencial tóxico, mutagênico, carcinogênico e habilidade de se acumular na cadeia trófica.

Segundo o relatório apresentado em 2001 pela Agência Nacional de Águas (ANA), cerca de 70\% dos rios que fazem parte das bacias hidrográficas que vão do Sergipe ao Rio Grande do Sul apresentaram altos índices de contaminação, principalmente por efluentes urbanos, substâncias lixiviadas de grandes lixôes e agrotóxicos. Normalmente, uma importante parcela do processo de contaminação pode ser atribuída às atividades das refinarias de petróleo e seus derivados. O relatório da comissão mista para analisar o acidente na
Petrobrás/Repar cita 33 acidentes ocorridos com derramamento de petróleo e seus derivados, no Brasil, no período de 1975 a 2001, somando milhôes de litros que contaminaram solos, rios e mar. Um dos acidentes mais recentes ocorreu em julho de 2000, durante a operação de transferência de petróleo do terminal São Francisco do Sul, litoral de Santa Catarina, para a refinaria de Araucária. Cerca de 4 milhóes de litros de petróleo atingiram os rios Barigüi e Iguaçu. Após o evento, concentrações elevadas de benzeno, tolueno, etil-benzeno, xilenos e naftaleno foram detectadas, o que provocou significativos danos à flora e fauna aquáticas e sério risco à população humana ali residente (Tiburtius, Zamora e Leal, 2004).

A contaminação de águas subterrâneas por compostos orgânicos deste tipo representa sérios problemas à saúde pública. As espécies benzeno, tolueno e xilenos (BTXs) são freqüentemente encontradas em águas subterrâneas, por causa de vazamentos em tanques de estocagem. A Agência Ambiental Norte Americana (EPA) estima que existam mais de 1,5 milhões de tanques subterrâneos de armazenamento de gasolina nos Estados Unidos, nos quais semanalmente, são encontrados mais de mil novos vazamentos (Tiburtius, Zamora e Leal, 2004). No Brasil existem, aproximadamente, 27.000 postos de gasolina (Petrobrás, 1995), dos quais a grande maioria surgiu na década de 70 , quando houve um grande aumento do número de postos no país. Como a vida útil dos tanques de armazenamento é de aproximadamente 25 anos, pode-se esperar um grande aumento da ocorrência de vazamentos de contaminantes derivados de petróleo nos postos do país (Corseuil e Martins, 1997).
Os contaminantes que têm maior solubilidade em água, como hidrocarbonetos monoaromáticos, benzeno, tolueno, etilbenzeno e xilenos, são considerados os mais perigosos por serem substâncias depressoras do sistema nervoso central e podem também causar leucemia em exposiçōes crônicas(Tiburtius, Zamora e Leal, 2004).

O processo de contaminação do solo e das águas subterrâneas pode ser visualizado na Figura 1.

Primeiramente o poluente despejado na superfície do solo permeia por uma região insaturada do solo. Com o passar do tempo o poluente forma uma frente de avanço que pode chegar e se acumular na região saturada do solo ou atingir os rios (Bear, 1972).

As técnicas de remediação de sítios contaminados são consideradas caras e, portanto ainda pouco praticadas (Lee e Lin, 2000). Em alguns casos a remediação é desnecessária, uma vez que a degradação natural dos compostos (reação bioquímica mediada por microorganismos que "consomem" o poluente) pode impedir a contaminação de águas efetivamente utilizadas por populações, ou ainda, as atividades de remediação de solos e aqüíferos podem ser realizadas em posiçóes e tempos inapropriados devido à falta de conhecimento da distribuição espacial do poluente. Por isso surge a necessidade de ferramentas que auxiliem na tomada de decisões, quanto a açôes corretivas em face de vazamentos iminentes. Seria interessante prever a profundidade atingida pela substância no solo em função do tempo de contato, assim como a distribuição da concentração da substância em diferentes pontos do solo. Isto permitiria estimar o dano

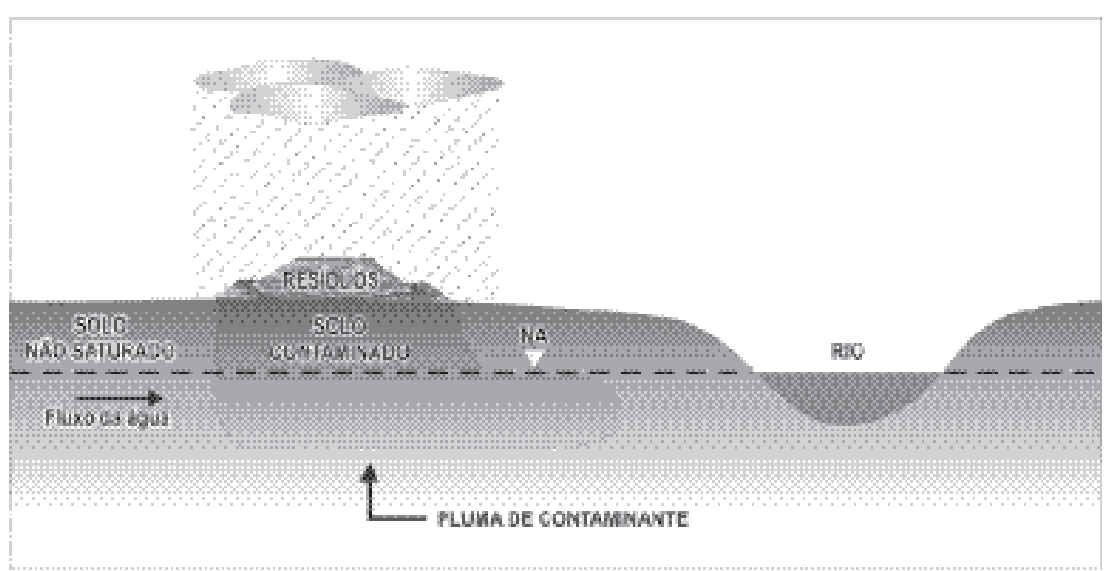

Figura I - Processo de contaminação do solo 
ambiental causado por algum acidente, estudar o tempo de residência no solo de diferentes poluentes, ajudar no desenvolvimento e implementação de técnicas de remediação do solo e águas, entre outras possibilidades.

Desenvolveu-se assim, um código computacional para resolver o modelo matemático de transporte de poluentes bidimensional em meios porosos com a finalidade de se fazer simulações da dispersão de poluentes em solos, permitindo assim, fazer as predições citadas anteriormente.

\section{METODOLOGIA}

\section{Modelo matemático}

Os principais processos de transporte de poluentes em solos incluem advecção e a dispersão (dispersão hidrodinâmica + difusão molecular) (Alshawabkeh, Rahbar e Sheahan, 2005).

Advecção é o movimento de contaminantes causado pelo escoamento de um fluido através do meio poroso. Assim, este fluido que se move com certa velocidade acaba por transportar o contaminante consigo (Bear, 1972). A velocidade de transporte é função da velocidade de Darcy $V\left[\mathrm{LT}^{-1}\right]$ do meio poroso e pode ser representada pela Equação 1 .

$V=-\frac{K}{\mu} \frac{\partial P}{\partial x}$

A velocidade intersticial $V_{i}\left[\mathrm{LT}^{-1}\right]$ no meio poroso, representada pela Equação 2, é igual a velocidade de Darcy $(V)$ dividida pela porosidade efetiva $n$ [-].

$V_{i}=-\frac{K}{n \mu} \frac{\partial P}{\partial x_{i}}$

Ainda, $K\left[\mathrm{LT}^{-1}\right]$ é a condutividade hidráulica, $\mu\left[\mathrm{ML}^{-1} \mathrm{~T}^{-1}\right]$ é a viscosidade do fluido, $P\left[\mathrm{ML}^{-1} \mathrm{~T}^{-2}\right]$ é a pressão e $x_{i}[L]$ é a distância longitudinal nesta direção. O fluxo advectivo de massa é dado pela Equação 3:

$\mathrm{fa}_{\mathrm{x}}=\mathrm{V}_{\mathrm{C}} \mathrm{C}$

onde $f a_{x}\left[\mathrm{MT}^{-1} \mathrm{~L}^{-2}\right]$ é o fluxo advectivo e $C\left[\mathrm{ML}^{-3}\right]$ é a concentração do poluente.

Já a difusão é um processo de transporte molecular de massa no qual o soluto move-se de regiōes de maior concentração para regiōes de menor concentração. $\mathrm{O}$ transporte no subsolo devido à difusão, pode ser descrito pela primeira Lei de Fick da difusão, dada pela equação 4 (Bird et al, 2003):
$\mathrm{fd}_{\mathrm{x}}=-\mathrm{D} \frac{\partial \mathrm{C}}{\partial \mathrm{x}_{\mathrm{i}}}$

onde $f d_{x}\left[\mathrm{MT}^{-1} \mathrm{~L}^{-2}\right]$ é o fluxo difusivo de massa na direção $i, D\left[\mathrm{~L}^{2} \mathrm{~T}^{-1}\right]$ é o coeficiente de dispersão e $d C / d x_{i}\left[\mathrm{ML}^{-4}\right]$ é o gradiente de concentração nesta mesma direção.

O ponto inicial no desenvolvimento de equações diferenciais para descrever o transporte de solutos em meios porosos é considerar a conservação de soluto em um volume elementar fixo no domínio do escoamento (Welty, Wicks e Wilson, 1984). Em forma literal, esta equação é dada pela Equação 5:

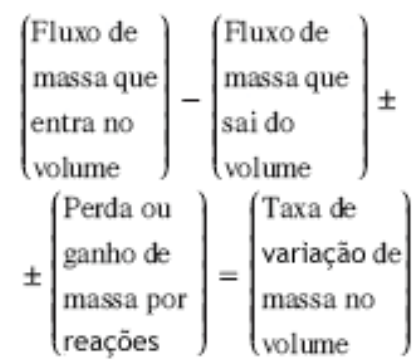

Os processos físicos que controlam o fluxo de massa que entra e sai do volume elementar são advecção e a dispersão hidrodinâmica. A perda ou ganho de massa de soluto no volume elementar pode ocorrer como um resultado de processos físicos, químicos e biológicos.

Neste trabalho não foram considerados os processos de perda e ganho de massa. Desse modo o modelo representa os processos de transporte por advecção e dispersão.

Dito isto, a equação diferencial que descreve o transporte de poluentes em um meio poroso é dada pela Equação 6:

$\frac{\partial}{\partial \mathrm{t}}(\rho \mathrm{C})=\frac{\partial}{\partial \mathrm{x}_{\mathrm{i}}}\left(\rho \mathrm{D} \frac{\partial \mathrm{C}}{\partial \mathrm{x}_{\mathrm{i}}}\right)-\frac{\partial}{\partial \mathrm{x}_{\mathrm{i}}}\left(\alpha \rho \mathrm{C} \mathrm{V}_{\mathrm{i},}\right.$

Onde a primeira parcela do lado direito da equação 6 é a contribuição difusiva e a segunda parcela é a contribuição advectiva (Mironenko e Pachepsky, 1998). Diferentes tipos de solos influenciam os parâmetros coeficiente de dispersão $(D)$ e a velocidade intersticial $(V)$. Assim, solos heterogêneos também podem ser representados por este modelo, porém devem-se usar esses parâmetros de forma coerente para cada camada de solo.

\section{Metodologia numérica}

O método adotado para a discretização da equação diferencial do modelo foi o dos volumes finitos (Maliska, 1995), com interpolação de segunda ordem usando diferenças centrais. O método dos volumes finitos consiste em obter a aproximação numérica da equação diferencial a partir de sua integração no volume de controle elementar que está representado pela Figura 2. A discretização é feita integrando todos os termos da equação que governa o fenômeno no espaço e no tempo para cada volume de controle do domínio. Como resultado tem-se a equação discretizada contendo os valores da grandeza de interesse para um grupo de pontos de uma malha.

Portanto, integrando a Equação 6 no volume em duas dimensões ( $\mathrm{x}$ e y) conforme mostrado na Figura 2, é obtida a Equação 7.

$$
\begin{aligned}
& \int_{w}^{e} \int_{s}^{n}\left[\frac{\partial}{\partial x}\left(\rho \mathrm{D} \frac{\partial \mathrm{C}}{\partial \mathrm{x}}\right)-\frac{\partial}{\partial \mathrm{x}}\left(\rho \mathrm{CV}_{\mathrm{x}}\right)\right] \mathrm{dxdy}+ \\
& +\int_{\mathrm{w}}^{e} \int_{s}^{\mathrm{u}}\left[\frac{\partial}{\partial \mathrm{y}}\left(\rho \mathrm{D} \frac{\partial \mathrm{C}}{\partial \mathrm{y}}\right)-\frac{\partial}{\partial \mathrm{y}}\left(\rho \mathrm{CV}_{y}\right)\right] \mathrm{dxdy} \\
& =\int_{\mathrm{w}}^{e} \int_{\mathrm{s}}^{\mathrm{n}}\left(\frac{\partial}{\partial \mathrm{t}}(\rho \mathrm{C})\right) \mathrm{dxdy}
\end{aligned}
$$

Ao integrar a equação de conservação chega-se na Equação 8, em termos dos fluxos difusivos e advectivos em cada face do volume:

$$
\begin{aligned}
& {\left[\left(\rho \mathrm{D} \frac{\partial \mathrm{C}}{\partial \mathrm{x}}\right)\left|=-\left(\rho \mathrm{D} \frac{\partial \mathrm{C}}{\partial \mathrm{x}}\right)\right|_{w}-\right.} \\
& \left.-\rho \mathrm{V}_{\mathrm{x}} \mathrm{Cl}+\rho \mathrm{V}_{\mathrm{x}} \mathrm{Cl}_{\mathrm{w}}\right] \Delta \mathrm{y}+ \\
& +\left[\left.\left(\rho \mathrm{D} \frac{\partial \mathrm{C}}{\partial \mathrm{y}}\right)\right|^{\mathrm{a}}-\left.\left(\rho \mathrm{D} \frac{\partial \mathrm{C}}{\partial \mathrm{y}}\right)\right|_{s}-\rho \mathrm{V}_{y} \mathrm{C}\right)+ \\
& \left.+\rho \mathrm{V}_{\mathrm{y}} \mathrm{Cl}\right] \Delta \mathrm{x} \\
& =\frac{\partial}{\partial \mathrm{t}}(\rho \mathrm{C}) \Delta \mathrm{x} \Delta \mathrm{y}
\end{aligned}
$$

Estes fluxos podem ser calculados em termos dos valores da variável avaliados nos centros dos volumes. Para a aproximação dos fluxos difusivos foram utilizadas diferenças centrais de $2^{\text {a }}$ ordem. Seguindo a notação mostrada na Figura 2, tem-se:

$\left.\frac{\partial \mathrm{C}}{\partial \mathrm{x}}\right|_{e}=\frac{\mathrm{C}_{\mathrm{E}}-\mathrm{C}_{\mathrm{p}}}{\Delta \mathrm{x}_{\mathrm{e}}},\left.\frac{\partial \mathrm{C}}{\partial \mathrm{X}}\right|_{\mathrm{w}}=\frac{\mathrm{C}_{\mathrm{p}}-\mathrm{C}_{\mathrm{w}}}{\Delta \mathrm{x}_{\mathrm{W}}}$,
$\left.\frac{\partial \mathrm{C}}{\partial \mathrm{Y}}\right|_{\mathrm{a}}=\frac{\mathrm{C}_{\mathrm{N}}-\mathrm{C}_{\mathrm{p}}}{\Delta \mathrm{y}_{\mathrm{n}}}+\left.\frac{\partial \mathrm{C}}{\partial \mathrm{y}}\right|_{\mathrm{s}}=\frac{\mathrm{C}_{\mathrm{p}}-\mathrm{C}_{\mathrm{S}}}{\Delta \mathrm{y}_{\mathrm{s}}}$

Para os fluxos advectivos nas faces dos volumes, usando também diferenças centrais:

$\left.C\right|_{0}=\frac{C_{E}+c_{\mathrm{P}}}{2},\left.C\right|_{w}=\frac{C_{\mathrm{P}}+C_{W}}{2}$,
$\left.C\right|_{\mathrm{u}}=\frac{\mathrm{C}_{\mathrm{N}}+\mathrm{C}_{\mathrm{P}}}{2}+\left.\mathrm{C}\right|_{\mathrm{s}}=\frac{\mathrm{C}_{\mathrm{P}} \mathrm{C}_{\mathrm{s}}}{2}$ 


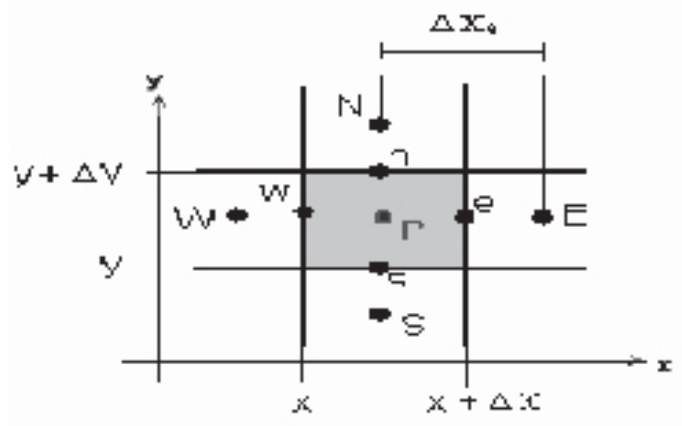

Figura 2 - Volume de controle elementar e seus vizinhos para o caso bidimensional

Desta forma, após substituir as expressóes para os fluxos difusivos e advectivos (Equações 9 e 10) na Equação de conservação (Equação 8), tem-se:

$$
\begin{aligned}
& \frac{\partial}{\partial \mathrm{t}} \Delta \mathrm{x} \Delta \mathrm{y}=\mathrm{A}_{\mathrm{e}} \mathrm{C}_{\mathrm{E}}+\mathrm{A}_{\mathrm{w}} \mathrm{C}_{\mathrm{W}}+\mathrm{A}_{\mathrm{n}} \mathrm{C}_{\mathrm{N}}+ \\
& +\mathrm{A}_{\mathrm{s}} \mathrm{C}_{\mathrm{S}}-\mathrm{A}_{\mathrm{p}} \mathrm{C}_{\mathrm{F}}
\end{aligned}
$$

Onde os coeficientes Ai são definidos por:

$$
\begin{aligned}
& \mathrm{A}_{\mathrm{e}}=\left(\frac{\rho \mathrm{D}}{\Delta \mathrm{x}_{\mathrm{e}}}-\frac{\rho \mathrm{V}_{\mathrm{e}}}{2}\right) \Delta \mathrm{y}, \\
& \mathrm{A}_{\mathrm{w}}=\left(\frac{\rho \mathrm{D}}{\Delta \mathrm{x}_{\mathrm{w}}}+\frac{\rho \mathrm{V}_{\mathrm{w}}}{2}\right) \Delta \mathrm{y} \\
& \mathrm{A}_{\mathrm{p}}=\mathrm{A}_{\mathrm{e}}+\rho \mathrm{V}_{\mathrm{e}} \Delta \mathrm{y}+\mathrm{A} \\
& \mathrm{w}_{\mathrm{w}}-\rho \mathrm{V}_{\mathrm{w}} \Delta \mathrm{y}+\mathrm{A}_{\mathrm{n}}+
\end{aligned}
$$

$\mathrm{A}_{\mathrm{p}}=\mathrm{A}_{\mathrm{e}}+\rho \mathrm{V}_{\mathrm{e}} \Delta \mathrm{y}+\mathrm{A}_{\mathrm{w}}-\rho \mathrm{V}_{\mathrm{w}} \Delta \mathrm{y}+$

$A_{\mathrm{n}}+\rho \mathrm{V}_{\mathrm{n}} \Delta \mathrm{x}+\mathrm{A}_{\mathrm{s}}-\rho \mathrm{V}_{\mathrm{s}} \Delta \mathrm{x}$

Nos contornos do domínio, a equação 8 deve ser avaliada individualmente (Maliska, 1995). A velocidade $(V i)$ e o coeficiente de dispersão $(D)$ são parâmetros de entrada característicos do poluente e do tipo de solo em questão. O sistema de equações diferencias ordinárias resultante é resolvido pelo método das linhas (Davis, 1984). Para cada simulação é necessário, portanto, definir as propriedades de transporte e a concentração inicial do poluente ao longo do domínio.

\section{Implementação computacional}

O código computacional para simulação do modelo proposto foi implementado em linguagem C. Foi usado um pacote numérico de código aberto, chamado Dasslc (Dasslc, 1997), para resolução do sistema de equações diferenciais ordinárias resultantes da discretização espacial da equação do modelo. As outras ferramentas usadas no trabalho foi o software lcc-win32 como Editor/compilador (Lcc-win32, 2005) e os softwares Mayavi (Mayavi, 2005) e Gnuplot (Gnuplot, 1993) para visualização dos resultados simulados. Desse modo, todos os softwares utilizados neste trabalho são livres.

\section{RESULTADOS E DISCUSSÃO}

\section{Validação numérica}

Para validar o código numérico, utilizaram-se comparaçôes com a solução analítica unidimensional para difusão/advecção (Cremasco, 2003). Assim, foram utilizados diferentes tipos de malhas com modificação no número de volumes $(N x)$. Na Figura 3 apresenta-se a comparação da solução numérica com a analítica para difusão pura usando-se uma malha com $N x$ igual 50. As condições e parâmetros do problema foram: Comprimento $(L)=70 \mathrm{~m}$ e coeficiente de difusão $(D)=0.1 \mathrm{~m}^{2} / \mathrm{s}$. A concentração inicial foi considerada nula $(C(x, 0)=0.0)$ $\mathrm{e}$ as condiçôes de fronteira foram de concentração adimensional igual a um $(C=1.0)$ em uma extremidade e fluxo mássico nulo na outra extremidade.

Os resultados estão ilustrados pela Figura 3 onde é feita uma comparação da solução analítica com a numérica em três intervalos de tempo. A partir dos resultados mostrados percebe-se que o método numérico apresenta uma boa precisão para o caso de difusão pura.

$\mathrm{Na}$ Figura 4 ilustra-se o gráfico da comparação da solução numérica com a analítica para o processo de difusão/ advecção.
As condições de contorno foram iguais ao caso da difusão pura e a velocidade intersticial $(V)$ foi de $0.1 \mathrm{~m} / \mathrm{s}$. A partir dos resultados mostrados pela figura 4 percebe-se que o método numérico também apresenta uma boa precisão para o caso de transporte por difusão/advecção.

\section{Resultado numérico versus experimental}

Foram realizados experimentos em laboratório, referentes à dispersão de algumas substâncias em meios porosos. Estes experimentos consistiram em acompanhar de forma visual o avanço do poluente no meio em função do tempo de contato. Os resultados obtidos foram usados para comparação com resultados trazidos por simulaçôes realizadas com o código computacional desenvolvido.

Nas Figuras 5, 6 e 7 ilustramse os experimentos montados para acompanhar a dispersão de poluentes líquidos em areia. Buscou-se um experimento simples e de baixo custo que permitisse investigar a validade das predições trazidas pelas simulações numéricas. Neste trabalho, o poluente analisado foi gasolina por ser este um dos principais contribuintes do problema da contaminação de aquiíferos por BTXs. Foi utilizada areia como meio de transporte do poluente pelo fato de facilitar a visualização da região na qual o poluente está se propagando. Por estar seca, pôde-se perceber de forma visual a frente de avanço da pluma contaminante sem a adição de substâncias corantes. Poderia ter sido estudado outros meios porosos e/ou solos sob condições saturadas. Entretanto, isso dificultaria o acompanhamento do avanço da pluma contaminante de forma visual e, desta forma, técnicas de detecção mais elaboradas seriam necessárias. Deve se destacar que, do ponto de vista numérico, não haveria nenhum problema em lidar com estas situações.

Os aparatos experimentais mostrados nas Figuras 5 e 6 foram utilizados para o estudo da dispersão de poluentes em uma dimensão. Na Figura 7 é mostrado o experimento utilizado para o estudo do mesmo em duas dimensões.

Na Figura 5 ilustra-se um experimento que consiste num tubo de vidro vertical com duas aberturas, uma superior e uma inferior. $\mathrm{Na}$ abertura superior é colocado o poluente. Outra 


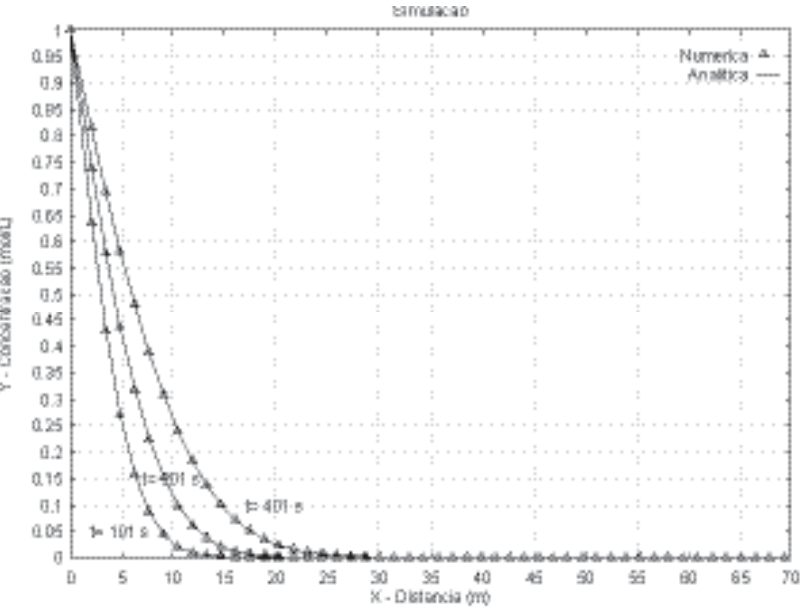

Figura 3 - Comparação da solução analítica e numérica para difusão pura com $\mathrm{Nx}=\mathbf{5 0}$

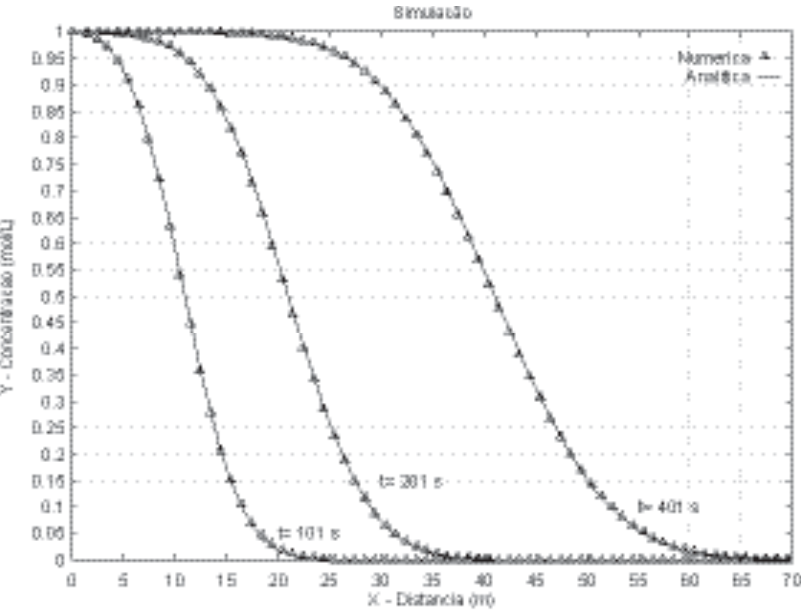

Figura 4 - Comparação da solução analítica e numérica para difusão/advecção com $\mathrm{Nx}=70$

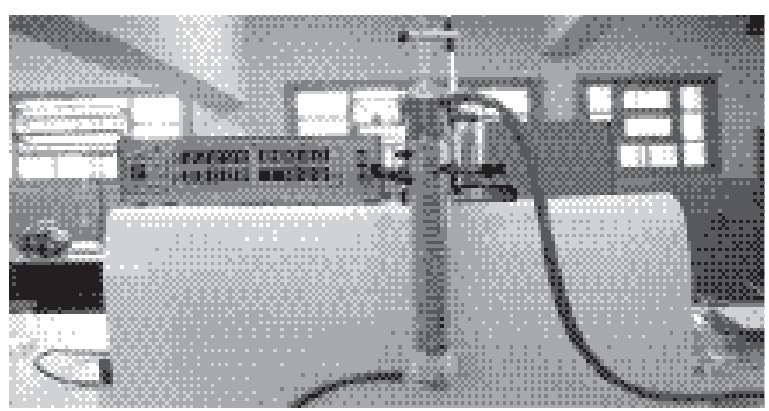

Figura 5 - Difusão e advecção unidimensional

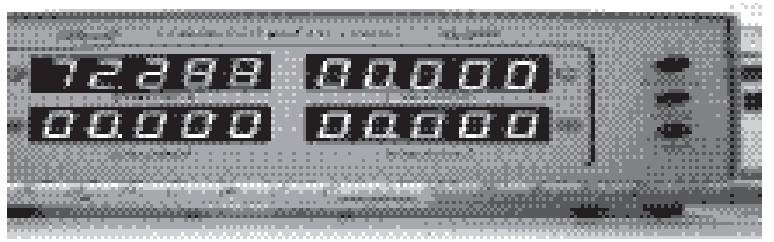

Figura 6 - Difusão unidimensional horizontal

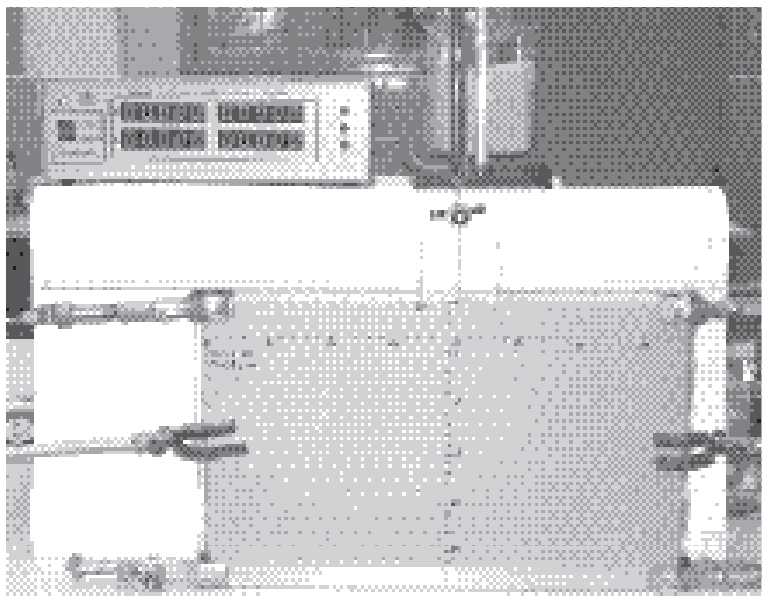

Figura 7 - Difusão e advecção bidimensional abertura lateral superior foi usada para garantir a manutenção do mesmo nível durante a execução do experimento. A abertura inferior permite a saída de ar contida nos poros sem afetar a migração do poluente. A evolução da frente de avanço em função do tempo foi registrada visualmente através de fotografias obtidas em seqüência.

$\mathrm{Na}$ figura 6 ilustra-se um experimento parecido ao da figura 5. A diferença é que o transporte de poluente se dá pela diferença de concentração, uma vez que o fluxo é horizontal e não existe a contribuição advectiva. Assim, o poluente entra por uma extremidade do tubo mostrado e avança devido à diferença de concentração.

$\mathrm{Na}$ figura 7 ilustra-se o transporte bidimensional, ou seja, o poluente é transportado no sentido vertical devido a advecção e a dispersão e no sentido lateral devido a dispersão somente. Nesse caso, utilizaram-se duas chapas paralelas de vidro, sendo preenchido de areia o volume entre estas elas.

Difusão e advecção unidimensional

A simulação do experimento da dispersão de poluentes unidimensional foi efetuada sob as condiçôes em que foram feitos os experimentos. As con-

dições foram as seguintes: $\boldsymbol{L}=\mathbf{0 , 3 4 m}$ (comprimento da coluna); $\boldsymbol{N} \boldsymbol{x}=\mathbf{5 0}$ (número de volumes na direção analisada); $D=5.6 \times 10^{6}\left[\mathrm{~m}^{2} / \mathrm{s}\right]$ (coeficiente de dispersão); $V=2,2 x 10^{-4}[\mathrm{~m} / \mathrm{s}]$ (velocidade intersticial); $\boldsymbol{C}(\boldsymbol{x}, \boldsymbol{0})=\boldsymbol{0 . 0}$ (concentração 
inicial). $\mathrm{O}$ coeficiente de dispersão e a velocidade intersticial foram obtidos a partir de estimativas realizadas com o próprio código computacional levando-se em conta os resultados obtidos nos experimentos. Como condição de contorno usou-se fluxo nulo em uma extremidade e concentração adimensional igual a um $(\boldsymbol{C}(\boldsymbol{0}, \boldsymbol{t})=\mathbf{1 . 0})$ em outra extremidade. $\mathrm{Na}$ figura 8 ilustra-se a comparação do experimental com a simulação computacional feita a partir do código desenvolvido. Uma importante observação a se fazer é que a pressão de líquido sob a coluna de a reia foi mantida constante a fim de não interferir nos resultados experimentais.

A unidade da escala mostrada ao lado das figuras gráficas é centímetros. Deve-se notar que a numeração na figura do experimento começa em 2 enquanto na figura gráfica começa em 0 . Assim, ao se comparar deve-se considerar essa defasagem.

A concentração decresce do preto para o branco, como ilustrado na Figura 9.

A partir da Figura 8 pode-se verificar que há uma boa concordância entre os resultados experimentais e os simulados e pode-se concluir que o modelo matemático descrito anteriormente conseguiu representar bem a frente de avanço do poluente.

Difusão e advecção bidimensional

Para a simulação da dispersão de poluentes bidimensional também se buscou entrar com as condiçôes em que o experimento ocorreu. Assim os seguintes parâmetros foram usados: $\boldsymbol{L}=\mathbf{0 , 2 8 m}$ (comprimento da cuba); $\boldsymbol{M}=\mathbf{0 , 3 8 5 m}$ (largura da cuba); $\boldsymbol{N} \boldsymbol{x}=\mathbf{3 0}$ (número de volumes na dire-çãox); $N y=30$ (número de volumes na direção y); $\boldsymbol{D}=5.6 \times 10^{-6}\left[\mathrm{~m}^{2} / \mathrm{s}\right]$ (coeficiente de dispersão); $V \boldsymbol{y}=2,2 \times 10^{-4}[\mathrm{~m} / \mathrm{s}]$ (velocidade intersticial em $y$ ); $\boldsymbol{V} \boldsymbol{x}=\mathbf{0 . 0}$ (velocidade intersticial em $x$ ); $\boldsymbol{C}(\boldsymbol{x}, \boldsymbol{0})=\boldsymbol{0 . 0}$ (concentração inicial). O coeficiente de dispersão e a velocidade foram obtidos a partir de estimativas como descrito no caso anterior. As condições de contorno estão representadas pela Figura 10, onde "q" é o fluxo mássico.

A Figura 11 trás uma comparação entre os resultados experimentais e os simulados para o caso de difusão e advecção bidimensional.
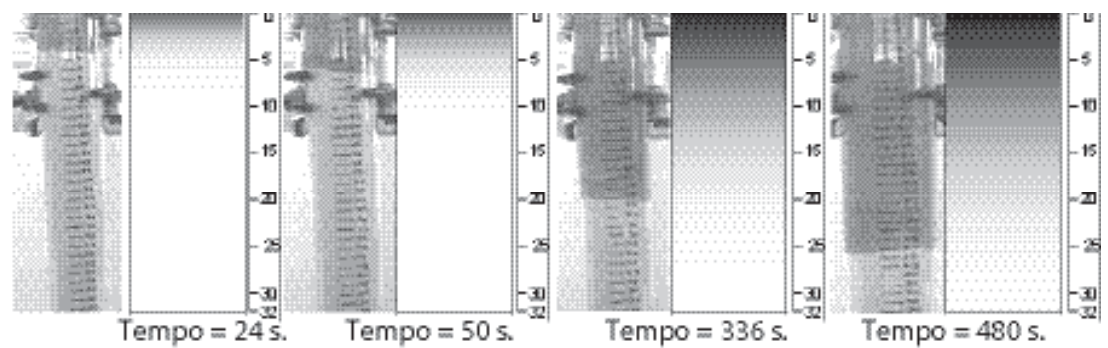

Figura 8 - Comparação do experimental versus numérico dos processos de difusão e advecção unidimensional

\section{$\begin{array}{lllllllll}0.000 & 0.142 & 0.285 & 0.427 & 0.570 & 0.712 & 0.855 & 0.997\end{array}$}

Figura 9 - Representação da escala de cores

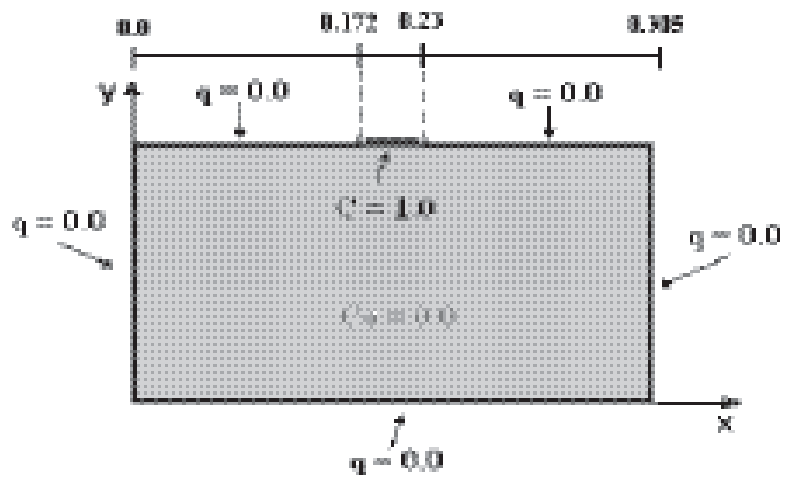

Figura 10 - Condição de contorno para difusão e advecção bidimensional
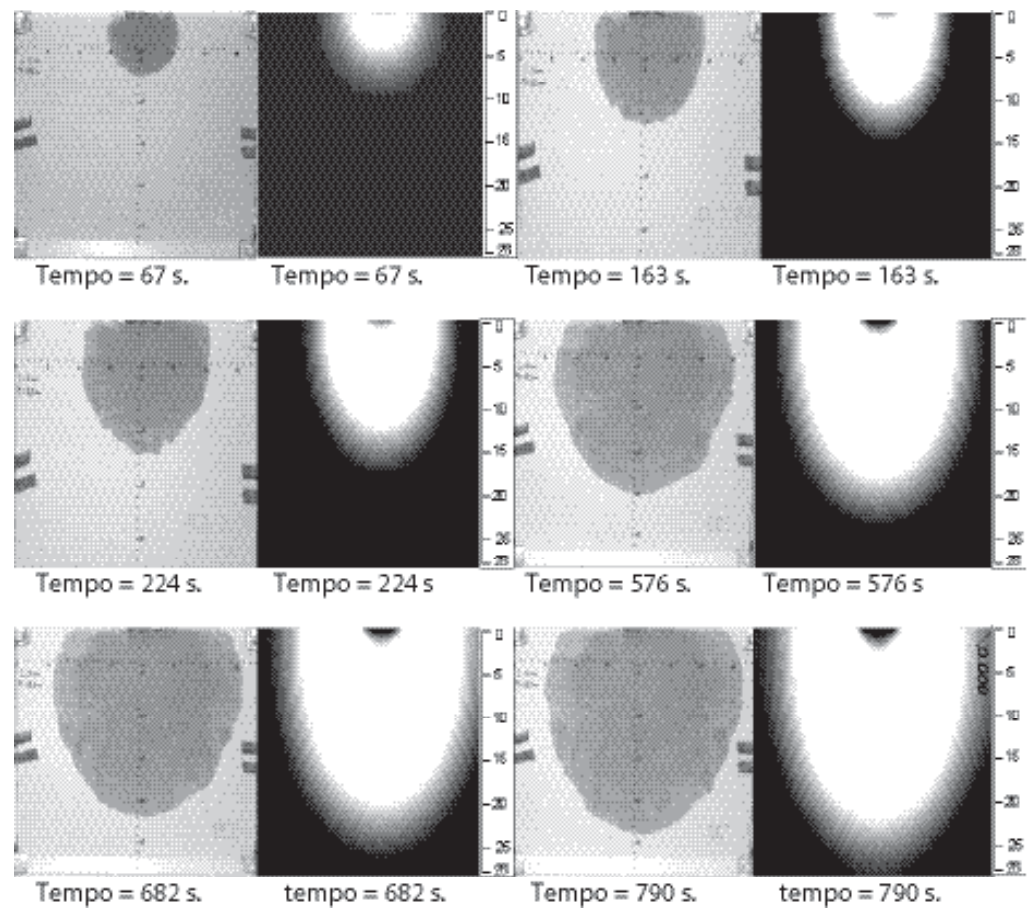

Figura I I - Comparação do experimental (esquerda) versus numérico (direita) de difusão e advecção bidimensional em solo arenoso para diversos tempos de contato de poluente 
A escala de cores está representada pela figura 12. Valores muito elevados de concentração (onde o poluente está sendo derramado) são encontrados na parte superior e é representado pelo lado direito da legenda. Valores com concentrações próximas a zero são obtidos nos lados e na parte inferior. Concentrações intermediárias são representadas pela cor branca

A partir da Figura 11 percebe-se que existe uma boa aproximação entre o resultado experimental e o simulado. A frente de avanço predita pela simulação acompanha muito bem a evolução daquela observada experimentalmente. Este exemplo mostra com clareza uma das possibilidades de aplicação do código desenvolvido, que é predizer a extensão da área afetada em um derramamento em escala maior, assim como os níveis de concentração do poluente, bastando para isso, conhecer os valores para as propriedades de transporte da substância no solo em questão.

\section{CONCLUSÃO}

Observou-se que os resultados obtidos numericamente têm boa concordância com os resultados experimentais. Pode-se dizer que as simulações levaram a boas predições do fenômeno em questão, representando de forma adequada a evolução da frente de avanço tanto para o caso unidimensional quanto para o caso bidimensional. A partir desses dados seria possível prever a dimensão da área contaminada por algum acidente e elaborar estratégias de remediação do local. Deve-se salientar que resultados mais precisos poderiam ser obtidos, contudo, seria necessário conhecer/estimar melhor as propriedades de transporte do meio. $\mathrm{O}$ código pode ser utilizado também na estimação de propriedades de transporte de diferentes poluentes em diferentes tipos de solos, parâmetros difíceis de serem encontrados na literatura.

Pretende-se agora incluir ao modelo os fenômenos de sorção e degradação e trabalhar mais a parte experimental. Foi feito também uma interface gráfica para que o software seja facilmente manipulado por qualquer pessoa que deseje fazer simulações como as apresentadas.

\section{AGRADECIMENTOS}

Agradecemos à Profa . Dr $^{\mathrm{a}}$. Eliane Endres e à Profa. Dra ${ }^{\mathrm{a}}$. Maria Helena

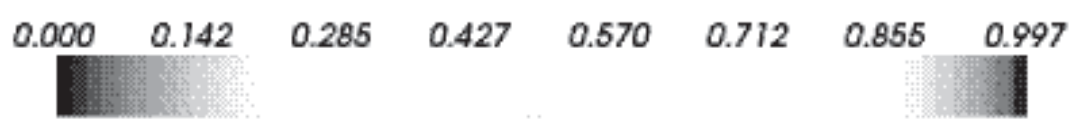

Figura I 2 - Representação da escala de cores

Comerlato pela colaboração dada na realização do artigo.

\section{REFERÊNCIAS}

ALSHAWABKEH, N. A.; RAHBAR, N.; SHEAHAN, T. A model for contaminant mass flux in capped sediment under consolidation. In: Journal of contaminant hydrology, (2005).

ANA - Relatório da Agência Nacional de Águas, 2001. Disponível em: http://www.ana.gov.br/diadaagua/relatorios.pdf. Acesso em 15 out. 2005.

BEAR, J. Dynamics of Fluids in Porous Media. Dover Publications, Inc.. New York, 1972.

BIRD, R. B; STEWART, W. E; LIGHTFOOT, E. N. Transport phenomena. 2nd edition, John Wiley and Sons, Nova York, 2003.

CORSEUIL, H. X.; MARTINS, M. D. M. Contaminação de Águas Subterrâneas por Derramamentos de Gasolina: O Problema é Grave? In: Engenharia Sanitária e Ambiental, Vol. 2, no. 2, Abril/Junho, 1997.

CREMASCO, M. A. Fundamentos de transferência de massa. 2a ed.: Editora da Unicamp, Campinas, SP, 729 p. 2003.

DASSLC - Differential Algebraic System Solver. Versão 2.0, 1997. Disponível em: http://www. enq.ufrgs.br/enqlib/numeric/numeric.html. Acesso em: 04 fev. 2006.

DAVIS, E. M. Numerical Methods and Modeling for Chemical Engineers. John Wiley \& Sons, New York, 1984

EDWARDS, C. A. Assessing the effects of environmental pollutants on soil organisms, communities, processes and ecosystems. In: European Journal of Soil Biology 38, (2002) p. 225-231.

GNUPLOT - A command-driven interactive function plotting program. Version 3.5, Aug/1993. Disponível em: http://www.gnuplot.info/. Acesso em: 12 fev. 2004

LCC-Win32. A free compiler system for windows operating systems by Jacob Navia. Version 3.3, May/2005. Disponível em: http://www. cs.virginia.edu/-lcc-win32/. Acesso em: 12 jun. 2005.

LEE, C.C.; LIN, S. D. Handbook of environmental engineering calculations. New York: McGraw-Hill, 2000. p. $1223-1261$.

MALISKA, C. R. Transferência de Calor e Mecânica dos fluidos computacional. Fundamentos e coordenadas generalizadas. LTC editora, RJ, 424 p. 1995.

MAYAVI - The mayavi data visualizer - A free, powerful, scientific data visualiser written in python. Version 1.4, 2000-2005. Disponível em: http:// mayavi.sourceforce.net. Acesso em: 01 out. 2005.

MESQUITA, A. C. Uso das técnicas de oxidação química e biodegradação na remoção de alguns com- postos recalcitrantes. Tese - Universidade Federal do Rio de Janeiro, COPPE. Rio de Janeiro, 2004.

MIRONENKO, E. V.; PACHEPSKY, Ya. A. A. Estimating transport of chemical from soil to ponding water. In: Journal of hydrology 208, p. 53-61.(1998)

PETROBRÁS. Relatório Anual de Atividades. Rio de Janeiro, 1995.

RAJASEKARAN, G. et al. A. Contaminant transport modeling in marine clays. In: Ocean Engineering 32, (2005), p. 175-194.

REBOUÇAS, A. C. Caderno Técnico. ABAS, no 3, 1994.

TIBURTIUS, E. R. L.; PERALTA-ZAMORA, P. P.; LEAL, E. S. Contaminação de águas por $B T X$ s e processos utilizados na remediação de sítios contaminados. Quím. Nova, Vol.27, no 3, São Paulo, may/June 2004,

WELTY, J. R; WICKS, C. E; WILSON, R. E. Fundamentals of momentum, heat and mass transfer. 3rd ed.: John Wiley and Sons, Nova York, 1984.

Endereço para correspondência:

\author{
Jovani Luiz Favero \\ Universidade Estadual do Rio \\ Grande do Sul \\ Núcleo de Caxias do Sul \\ Av. Júlio de Castilhos, 3947 - 20 \\ andar \\ 950 I0-005 Caxias do Sul - RS \\ - Brasil \\ Tel.: (54) 3225-7486. \\ Email: favero@enq.ufrgs.br
}

